\title{
The Utility of DiceCT Imaging for High-Throughput Comparative Neuroanatomical Studies
}

\author{
Paul M. Gignac ${ }^{a}$ Nathan J. Kley ${ }^{b}$ \\ a Department of Anatomy and Cell Biology, Oklahoma State University Center for Health Sciences, Tulsa, OK, USA; \\ ${ }^{b}$ Department of Anatomical Sciences, Stony Brook University, Stony Brook, NY, USA
}

\section{Keywords}

Alcoholic iodine · Brain · DiceCT · Gray matter · Lugol's iodine $\cdot$ Myelin $\cdot$ Neuroanatomical imaging $\cdot$ Radiological contrast agents $\cdot$ White matter $\cdot \mathrm{X}$-ray micro-CT scanning

\begin{abstract}
Advancements in imaging techniques have drastically improved our ability to visualize, study, and digitally share complex, often minute, anatomical relationships. The recent adoption of soft-tissue $\mathrm{X}$-ray imaging techniques, such as diffusible iodine-based contrast-enhanced computed tomography (diceCT), is beginning to offer previously unattainable insights into the detailed configurations of softtissue complexes across Metazoa. As a contrast agent, dissolved iodine diffuses deeply throughout preserved specimens to bind fats and carbohydrates that are naturally present within metazoan soft tissues, increasing the radiodensities of these tissues in predictable ways. Like the current "gold standard" of magnetic resonance imaging, diceCT does not require physical dissection and can differentiate between the lipid content of myelinated versus nonmyelinated tissues, thereby offering great potential for neuroanatomical studies. Within the brain, for example, diceCT
\end{abstract}

distinguishes myelinated fiber tracts from unmyelinated cortices, nuclei, and ganglia and allows three-dimensional visualization of their anatomical interrelationships at previously unrealized spatial scales. In this study, we illustrate the utility of diceCT for the rapid visualization of both external and internal brain anatomy in vertebrates - alongside the intact bones of the skull and the complete, undisturbed pathways of peripheral nerves, up to and including the target organs that they innervate. We demonstrate the transformative potential of this technique for developing highresolution neuroanatomical datasets and describe best practices for imaging large numbers of specimens for broad evolutionary studies across vertebrates.

$$
\begin{aligned}
& \text { ๑) } 2018 \text { The Author(s) } \\
& \text { Published by S. Karger AG, Basel }
\end{aligned}
$$

\section{Introduction}

Over the last half century, nondestructive, three-dimensional (3D) visualization techniques have radically altered the landscape of the anatomical sciences. Tools such as X-ray computed tomography (CT), magnetic resonance imaging (MRI), selective plane illumination microscopy (SPIM), light sheet fluorescence microscopy

\begin{tabular}{ll}
\hline KARGER & $\begin{array}{l}\text { (c) } 2018 \text { The Author(s) } \\
\text { Published by S. Karger AG, Basel }\end{array}$ \\
E-Mail karger@karger.com & This article is licensed under the Creative Commons Attribution- \\
www.karger.com/bbe & NonCommercial-NoDerivatives 4.0 International License (CC BY- \\
NC-ND) (http://www.karger.com/Services/OpenAccessLicense). \\
Usage and distribution for commercial purposes as well as any dis- \\
tribution of modified material requires written permission.
\end{tabular}


Fig. 1. Comparison of human brains visualized using standard medical-grade MRI and diceCT technologies with details of the cerebellum, including the arbor vitae, enlarged. MRI (T1 weighted) example has $937.5 \times 937.5 \times 500 \mu \mathrm{m}$ voxel dimensions, whereas the diceCT example has $63.0 \times$ $63.0 \times 63.0 \mu \mathrm{m}$ voxel dimensions. DiceCT provides substantially higher resolution than standard MRI scans for large, complex neural structures like the human brain, while also differentiating between its myelinated and nonmyelinated components.

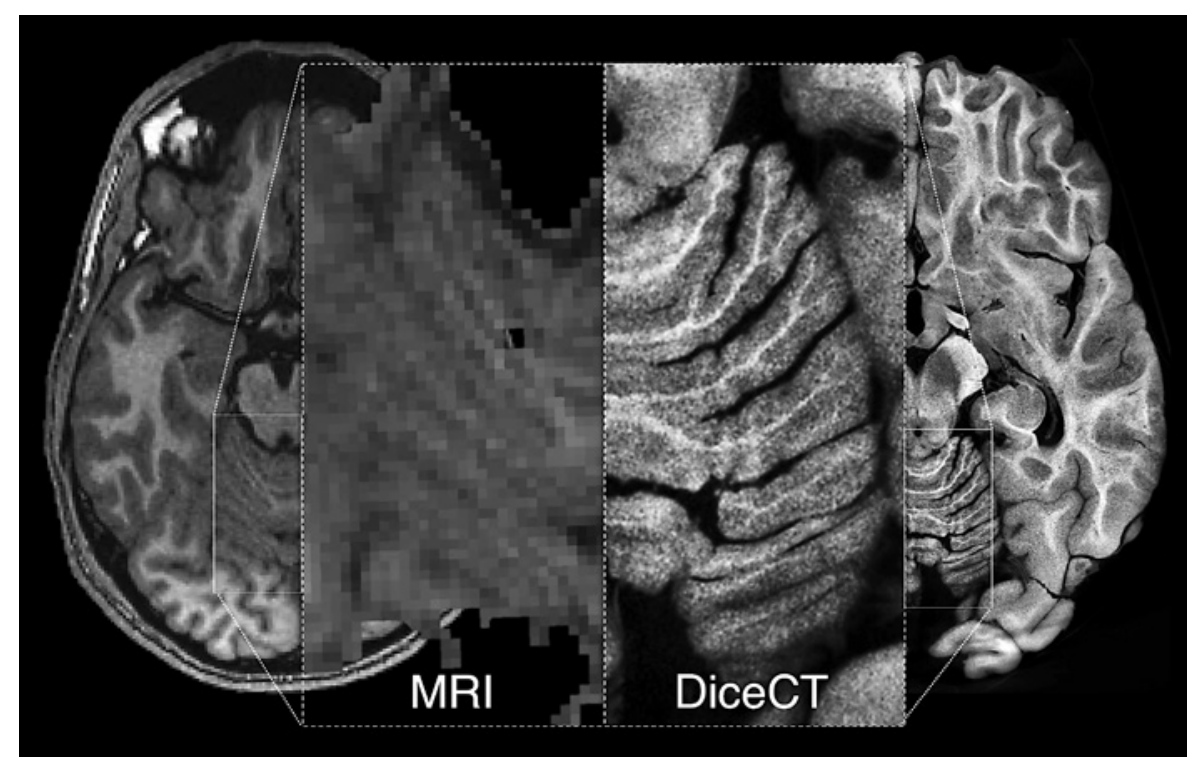

(LSFM), and optical projection tomography (OPT), among others, have greatly facilitated advances in quantifying the sizes, relationships, and spatial distributions of hard- and soft-tissue anatomical systems. These imaging modalities have given researchers newfound insights into neural systems, including the ability to rapidly document the detailed structure of the neurocranium (e.g., X-ray $\mathrm{CT}$ ), register brain morphology to skull morphology (e.g., MRI), document neural connections (e.g., SPIM and LSFM), and visualize gene expression patterns (e.g., OPT). MRI, in particular, has distinguished itself as the "gold standard" for neuroanatomical imaging due to its ability to contrast neural tissues from surrounding cerebrospinal fluid and cranial elements, differentiate between myelinated and nonmyelinated neural structures, and serve as a platform for the integration of additional visualization modalities (e.g., functional MRI, positron emission tomography). Magnetic resonance imagers come in a variety of configurations, including those for visualizing the large brains of humans [van de Moortele et al., 2009; Lüsebrink et al., 2017] or the far smaller brains of model developmental organisms, such as embryonic and postnatal rodents [e.g., $\mu \mathrm{MRI}$; Schneider et al., 2004]. MRI approaches used for human medical applications tend to be limited to voxel dimensions ranging from 250 to $1,000 \mu \mathrm{m}$ (Fig. 1), whereas $\mu \mathrm{MRI}$ provides much higher spatial resolution (25-100 $\mu \mathrm{m}$ voxels). Although the finer scale associated with $\mu \mathrm{MRI}$ has the potential to open up whole new avenues for neuroanatomical research, it is a comparatively expensive technique for routine use, and specimen diameter is typically limited to just a few centimeters due to relatively small gantry apertures [de Crespigny et al., 2008].

In this paper, we discuss a relatively new entrant into the collective of advanced anatomical visualization tools: diffusible iodine-based contrast-enhanced computed tomography ("diceCT") [Gignac et al., 2016]. DiceCT is poised to continue the march of documenting and quantifying metazoan neuroanatomy at progressively finer resolutions and across larger spatial scales (Fig. 1), while increasing the pace of data collection and, thus, the evolutionary scope of organisms that can be readily studied (Fig. 2). This technique relies on mature and widely available X-ray CT and $\mu \mathrm{CT}$ technologies [Neu and Genin, 2014 ], capable of imaging a wide variety of objects at micron and submicron scales. Although excellent at visualizing mineralized vertebrate tissues such as bone, dentin, and enamel, X-ray technologies are comparatively poor at differentiating relatively low-density soft-tissue structures, such as the central and peripheral nervous systems. To overcome this obstacle, diceCT utilizes iodine in the form of aqueous iodine-potassium iodide (a.k.a., Lugol's iodine solution, strong iodine solution, or simply $\mathrm{I}_{2} \mathrm{KI}$ ) or alcoholic iodine $\left(\mathrm{I}_{2} \mathrm{E}\right.$ and $\mathrm{I}_{2} \mathrm{M}$, using ethanol and methanol, respectively, as solvents [Metscher 2009a, 2009b, 2013]) as a radiopaque contrast agent [Wallingford, 1953].

Modern approaches to utilizing iodine as a contrast agent for its tissue-specific affinities in combination with high-resolution $\mu \mathrm{CT}$ imaging were pioneered by de 


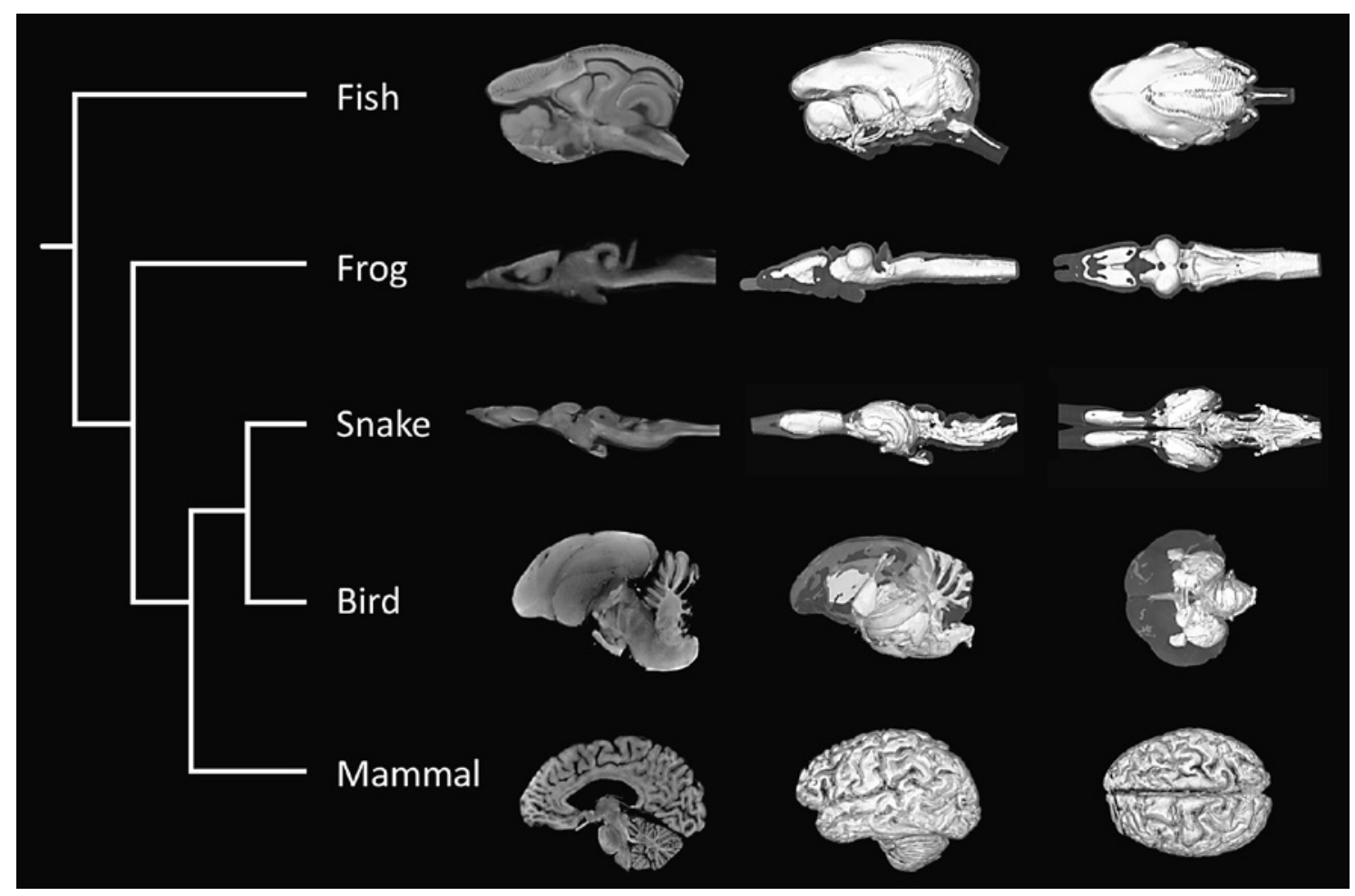

Fig. 2. Phylogenetic sample of diceCT-imaged brains, showing $2 \mathrm{D}$ parasagittal slices (left) together with $3 \mathrm{D}$ digital models of grayand white-matter structures in lateral (middle) and dorsal (right) projections. Gray matter is rendered as semitransparent, whereas white-matter tracts are rendered as opaque white. Representative taxa: Fish, Gnathonemus petersii; frog, Rana sylvatica [see Gignac et al., 2016]; snake, Liasis mackloti; bird, Sturnus vulgaris [see Gold et al., 2016]; mammal, Homo sapiens. All images and models are from adult specimens, span two orders of magnitude in brain size, and are not to scale. (Preservation, staining, and $\mu \mathrm{CT}$ imaging parameters available in online suppl. Table S4.)
Crespigny et al. [2008] and Metscher [2009a, b], with initial applications to vertebrate neuroanatomy and development. This pairing was quickly adopted by numerous other morphologists, who applied it to increasingly wider ranges of animal taxa and body sizes [Degenhardt et al., 2010; Herdina et al., 2010; Tobita et al., 2010; Tsai and Holliday, 2011; Gignac and Kley, 2014]. In 2015, various successful protocols for iodine-enhanced $\mu \mathrm{CT}$ imaging were formalized as "diceCT" by the Austin Working Group [Gignac et al., 2016]. To date, diceCT has been applied successfully to at least 12 classes of metazoans and has permitted the visualization of whole bodies and more restricted regions of interest across a 10,000-fold range in specimen sizes [see review in Gignac et al., 2016].

In solution, crystalized iodine forms into iodine polymers (e.g., triiodide, $\mathrm{I}_{3}^{-}$), which bind differentially to the lipids and carbohydrates that are naturally present within the soft tissues of animals (and plants). Once imbued, previously radiolucent soft tissues become radiodense, like their mineralized counterparts, causing them to absorb X-rays [Webb et al., 2005]. Different tissues contain different amounts of lipids and carbohydrates, making it possible to distinguish between bones, muscles, glands, fat bodies, epithelia, and nerves based on their affinities for iodine polymers and, thus, their radiodensities in Xray $\mu \mathrm{CT}$ images. Moreover, diceCT is particularly useful for visualizing central and peripheral nervous tissues because it allows for clear differentiation between myelinated and nonmyelinated structures [de Crespigny et al., 2008; Gignac and Kley, 2014]. This means that subtle tracts of white matter can be readily identified from surrounding gray matter within the brain [Gignac and Kley, 2014; Gignac et al., 2016] (Fig. 1-4; online suppl. Videos S1-S3; for all online suppl. material, see www.karger. com/doi/10.1159/000485476). Likewise, outside of the brain, myelinated peripheral-nerve processes can be distinguished from associated ganglia (Fig. 3; see online suppl. Videos S1-S3) [George and Holliday, 2013; Holli- 
day et al., 2013; Gignac and Kley, 2014]. Consequently, diceCT offers researchers the familiar, tissue-specific differentiation and larger scan volumes of MRI but at the finer resolutions of $\mu \mathrm{MRI}$ (and the lower costs of $\mu \mathrm{CT}$ ). DiceCT tissue contrasts are therefore achieved at 3D spatial scales unequaled by other current technologies and have the potential to visualize entire neural systems [Porro and Richards, 2017], from specific brain and spinalcord structures to the distal nerve processes and peripheral tissues they innervate.

Here, we demonstrate the potential for developing high-resolution neuroanatomical datasets and describe a pipeline to image large numbers of specimens for evolutionary studies across Vertebrata (Fig. 2). We discuss our experiences in preserving, staining, and $\mu \mathrm{CT}$ scanning more than 1,000 specimens in order to highlight the power of diceCT for capturing neuroanatomical details and the extent to which these can be rendered into $3 \mathrm{D}$ neuroanatomical models. Our goal is to outline how to begin using diceCT imaging for creating rich datasets as a neuroanatomy-specific supplement to the more generalized review provided by Gignac et al. [2016]. We do not anticipate that diceCT will replace standard histological approaches or 3D imaging modalities that have already become well-established methods throughout the neuroscience community. Instead, we hope that integrating diceCT into already existing neurovisualization workflows will provide for reciprocal illumination between structures and regions of interest, as well as increase the scientific value of research specimens, the quality of imaging data, and the ease of digitally sharing neuroanatomical resources - all of which have historically helped to advance the science.

\section{DiceCT Workflow}

Dozens of studies utilizing diceCT have been published over the last several years, and the variety of specimen types, histories, staining parameters, and scanning approaches used have informed on the best practices that are now recommended by veterans in the field [Gignac et al., 2016]. Here, we have built on the guidelines presented by Gignac et al. [2016] by taking an exploratory approach to optimizing diceCT for neuroanatomical visualizations across three primary methodological steps leading to successful data collection: (1) specimen preservation, (2) iodine staining, and (3) $\mu \mathrm{CT}$ imaging. We have also collated details of these steps from diceCT studies with already published neuroanatomical visualizations (31 publica-

DiceCT for 3D Neuroanatomical Imaging

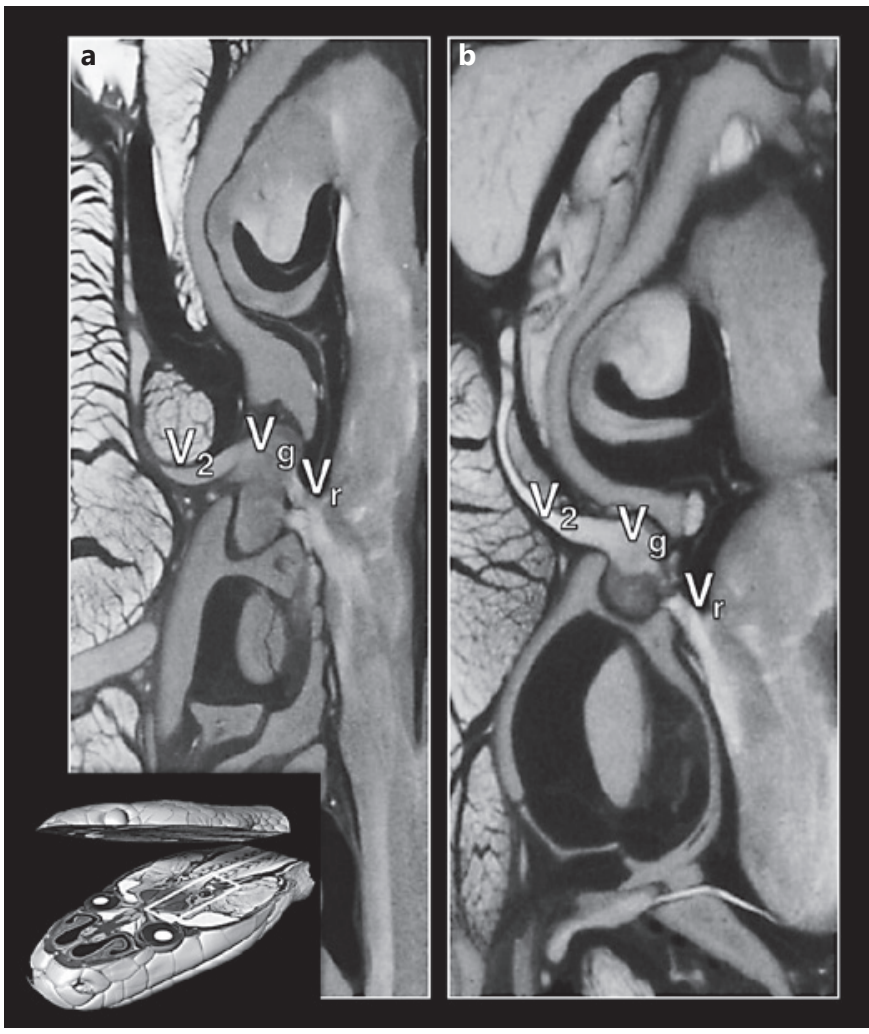

Fig. 3. $2 \mathrm{D}$ frontal slices through the left halves of the brains of two pythons, Liasis mackloti (a) and Antaresia maculosa (b), illustrating gray-scale differences between myelinated and nonmyelinated components of peripheral nerves (i.e., myelinated root $\left[\mathrm{V}_{\mathrm{r}}\right]$ ) and maxillary division $\left[\mathrm{V}_{2}\right]$ of the left trigeminal nerve versus the intervening nonmyelinated maxillomandibular ganglion $\left[\mathrm{V}_{\mathrm{g}}\right]$ ). Note also the numerous white-fiber tracts visible in adjacent portions of the (otherwise gray) brain. (Preservation, staining, and $\mu \mathrm{CT}$ imaging parameters are available in online suppl. Table S4.) The approximate locations of these slices are indicated by the white rectangle superimposed on the $3 \mathrm{D}$ reconstruction on the lower left (inset).

tions in total; see online suppl. Table S4). We end with a brief overview of data organization and analysis to help researchers exploit the full potential of diceCT datasets for producing 3D digital models like those presented here.

\section{Specimen Preservation}

Neural tissues are particularly sensitive to the means of preservation, and anatomy visualized via diceCT is only as clear as a specimen's initial (i.e., prestained) quality. Thus, great care should be taken in preparing specimens to ensure that diceCT data represent as accurately as possible the in vivo anatomy of the organism. All spec- 
imens must be chemically fixed, and we recommend that fixed tissues be as fresh as possible. (Access to fresh tissues for this study was carried out under guidelines established by the Stony Brook University Institutional Animal Care and Use Committee [protocol \#1726 to N.J.K. for Liasis mackloti and Antaresia maculosa] and the State Anatomical Board of Oklahoma [March 1, 2016, for Homo sapiens]; all other specimens were received as previously preserved.) Although numerous studies demonstrate research-quality visualizations using specimens not initially acquired or prepared specifically for diceCT imaging (i.e., vouchered museum specimens) [Herdina et al., 2010; Cox and Jeffery, 2011; Jeffery et al., 2011; Cox and Faulkes, 2014; Herdina et al., 2015a, b], the best contrasted - and, thus, easiest to model digitally - neural structures are those associated with specimens fixed immediately upon death [Hughes et al., 2016b; see also Fig. 1-4] (see online suppl. Videos S1-S3). We fix our specimens using 10\% phosphate-buffered formalin (a.k.a., neutral buffered formalin) or $4 \%$ paraformaldehyde, but numerous other fixatives can be effective as well (e.g., Bouin's solution and its alcoholic [DuboscqBrasil] variant, Dent's fixative, 70-95\% solutions of ethanol, or 2.5\% glutaraldehyde) [Gignac et al., 2016 and references therein].

Vouchered museum specimens represent appealing sources for the documentation of neuroanatomical variation. However, long-term storage of specimens in ethanol can limit the potential for tissue contrast. Fat is soluble in ethanol and other alcohols [Jeffery et al., 1989], and thus the lipids that are naturally present in myelinated nervous tissue can be leached out of these tissues over the course of decades in alcohol storage [e.g., Fig. 4 in Gignac et al., 2016]. Under these conditions, differentiation of white from gray matter becomes difficult, if not impossible, thereby limiting digital reconstructions to overall brain size and shape, without providing any details about the brain's internal structure. We do not recommend that researchers avoid using ethanol as a fixative or storage medium altogether. However, given the issue described above, we suggest that awareness can aid in choosing specimens (e.g., by avoiding particularly old, alcoholic specimens) that will maximize imaging success.

It is also common for opportunistically acquired specimens to be frozen for a period of time before being thawed prior to subsequent processing. Such specimens are generally to be avoided for diceCT imaging because the ice crystals that form during freezing can cause significant distortions within delicate soft tissues. In our experience, neural tissues are particularly susceptible to physical damage via the formation and subsequent growth of ice crystals. (These issues are readily apparent primarily as a direct result of the high spatial scale that diceCT affords.) Not only can frozen storage impact the fidelity of the anatomy itself, subsequently thawed brains also tend to contrast less well when stained with iodine.

It bears emphasis that field collection of specimens even without standard laboratory facilities - is compatible with sampling large numbers of specimens for high-throughput, comparative neuroanatomical studies, including those using diceCT. Hughes et al. [2016b] recently developed field fixation techniques that are effective for preserving neural tissues in remote conditions without laboratory facilities or cold storage. This approach uses both liquid (37\% volume/volume [v/v] formaldehyde) and solid (100\% formaldehyde powder) stocks to mix buffered formalin solutions in the field. The taxa collected by Hughes et al. [2016b] were chameleons from Uganda and the Democratic Republic of the Congo. Whole-body fixation was achieved via transcardial perfusion. Specimens were stored in a $10 \%$ buffered formalin solution containing $12 \%$ sucrose (weight by volume $[\mathrm{w} / \mathrm{v}]$ ) for up to 54 days in the field before reaching laboratory facilities [Hughes et al., 2016b]. (Note: Sucrose was added as a cryoprotectant in anticipation of future freezing upon return to the laboratory.) Specimens were subsequently prepared using Nissl staining, fluorescent immunohistochemical staining, and diceCT imaging. As a result of these efforts, there is a clear pathway to accessing potentially large numbers of specimens from remote locations for neuroanatomical research, expanding the potential breadth of comparative brain, spinal cord, and peripheral nerve studies [Striedter et al., 2014].

Neither Lugol's iodine nor X-ray imaging are new tools for anatomical researchers - it is in combination that they become uniquely powerful. Likewise, other well-established approaches can be harnessed alongside diceCT to ensure high-fidelity neuroanatomical data. One such technique is STABILITY. Modified from a hydrogel cross-linking procedure (CLARITY) [Chung et al. 2013], STABILITY was introduced by Wong et al. [2013] as a standardized, chemical polymerization process, in which a 3D meshwork of hydrogel infiltrates and physically reinforces whole specimens. The purpose of STABILITY is to limit size and shape distortions. This can be particularly useful for diceCT work, wherein exposure to high concentrations of iodine can cause unwanted distortions, such as shrinkage [Vickerton et al., 2013; Gignac and Kley, 2014; Gignac et al., 2016]. As demonstrated by Wong et al. [2013], and more recently by Carlisle et al. 
[2017], the reinforcing effects of STABILITY do not interfere significantly with iodine staining. When used together, it becomes possible to stain specimens at higher concentrations or durations than would otherwise be recommended because the mechanical reinforcement of soft tissues maintains their size and shape. STABILITY is implemented after fixation but before iodine staining and is effective for embryonic as well as postembryonic specimens [Wong et al., 2013; Carlisle et al., 2017]. Stabilized specimens can be destained and then prepared further using other staining techniques (as described below). (Note: Whether or not the STABILITY polymer can be depolymerized and fully removed from specimens has not been demonstrated to date.)

\section{Iodine Staining}

The three most commonly used forms of iodine for diceCT imaging include an aqueous iodine-potassium iodide $\left(\mathrm{I}_{2} \mathrm{KI}\right)$ solution (Lugol's solution), as well as two forms of alcoholic iodine, in which $\mathrm{I}_{2}$ crystals are dissolved directly into either $\sim 100 \%$ ethanol $\left(\mathrm{I}_{2} \mathrm{E}\right)$ or $\sim 100 \%$ methanol $\left(\mathrm{I}_{2} \mathrm{M}\right)$. Concentrations for these solutions are determined on a w/v basis. For example, a $1 \%$ aqueous solution of $\mathrm{I}_{2} \mathrm{KI}$ contains $1 \mathrm{~g}$ of solute (i.e., $0.33 \mathrm{~g} \mathrm{I}_{2}+0.67 \mathrm{~g}$ $\mathrm{KI})$ together with enough solvent (i.e., $\mathrm{H}_{2} \mathrm{O}$ ) to create 100 $\mathrm{mL}$ of staining solution; given the densities of $\mathrm{I}_{2}(4.933 \mathrm{~g} /$ $\mathrm{cm}^{3}$ ) and $\mathrm{KI}\left(3.123 \mathrm{~g} / \mathrm{cm}^{3}\right)$, this would equate with 99.72 $\mathrm{mL}$ of water in this particular instance. (Note: $\mathrm{KI}$ and $\mathrm{H}_{2} \mathrm{O}$ should be combined before adding $\mathrm{I}_{2}$ because the latter does not readily dissolve into an aqueous solution on its own.) Likewise, a $1 \%$ solution of $\mathrm{I}_{2} \mathrm{E}$ contains $1 \mathrm{~g}$ of $\mathrm{I}_{2}$ and $99.80 \mathrm{~mL}$ of $100 \%$ ethanol (which combine as $100 \mathrm{~mL}$ of total volume). Although not technically a percentage, the $\mathrm{w} / \mathrm{v}$ metric breaks down as how much mass of the staining agent is dissolved into a $100 \mathrm{~mL}$ volume (i.e., a proxy for "percentage") of staining solution. Online supplementary Document S5 describes the amounts of solute(s) and solvent needed to mix $1-10 \%$ solutions of $\mathrm{I}_{2} \mathrm{KI}, \mathrm{I}_{2} \mathrm{E}$, and $\mathrm{I}_{2} \mathrm{M}$. (The admixture for other concentrations can be calculated from the information provided.)

The two most important staining parameters are the concentration of the staining solution and the duration of time that the specimen remains in solution. Most diceCT specimens are successfully stained at fairly conservative concentrations of 1-10\% w/v [Gignac et al., 2016]. For a given specimen size, duration tends to be inversely related to concentration, such that lower-concentration solutions should be applied for longer periods of time and vice versa. In general, the smallest vertebrate specimens (e.g., embryos) stain overnight; many postembryonic ver- tebrates stain on the order of days to a few weeks; and larger adult specimens stain fully only after weeks to months [see Gignac et al., 2016, for a review on the relationships between specimen sizes, staining requirements, and requisite $\mathrm{CT}$ scanner beam energies]. Identifying the optimum level of staining is crucial to avoid either incomplete staining of specimens, which show uneven contrast levels within the same tissue type, or overstaining of specimens, which reduces the contrast between adjacent tissues [Gignac and Kley, 2014] and can lead to physical distortions [Vickerton et al., 2013; Wong et al., 2013; Carlisle et al., 2017].

Online supplementary Table S4 lists published diceCT studies that include neuroanatomical visualizations along with the types of specimens imaged in each. We recommend that users first identify the quality or type of specimens they would like to emulate from the literature and begin their initial staining based on the protocols described therein. From that point, researchers are able to increase or decrease exposure to iodine in subsequent specimens in order to produce the highest-quality visualizations for the questions being asked or hypotheses being tested.

\section{$\mu C T$ Imaging}

Imbuing soft tissues with iodine greatly increases their radiodensities. For example, thoroughly stained specimens may image in X-ray $\mu \mathrm{CT}$ scans with muscles, glands, and myelinated nervous tissues exhibiting greater radiodensities than cortical bone (Fig. 4a). As a result, the most consistent diceCT images are produced on $\mu \mathrm{CT}$ scanners that are also capable of generating high-quality skeletal or fossil datasets [Ketcham and Carlson, 2001; see also the review of CT scanner models and configurations in Gignac et al., 2016]. Embryonic and small postembryonic specimens can be imaged well with tabletop $\mu \mathrm{CT}$ systems, which typically have limited gantry sizes but sufficient beam energies. As specimen size increases, so does the volume of iodine needed to completely imbue the soft tissues of that specimen. As a result, most vertebrate specimens are best imaged using industrial-scale $\mu \mathrm{CT}$ scanners, which typically image volumes $\geq 100 \mathrm{~cm}^{3}$, are capable of beam energies beyond those commonly used to visualize cortical bone (i.e., $>10 \mathrm{~W}$ ), and have usercustomizable scanning configurations.

To produce high-quality neuroanatomical visualizations, we recommend that users set their $\mu \mathrm{CT}$ systems to image for several hours per specimen. Because iodinestained specimens are particularly dense and require higher-than-typical beam energies, there is potential for 


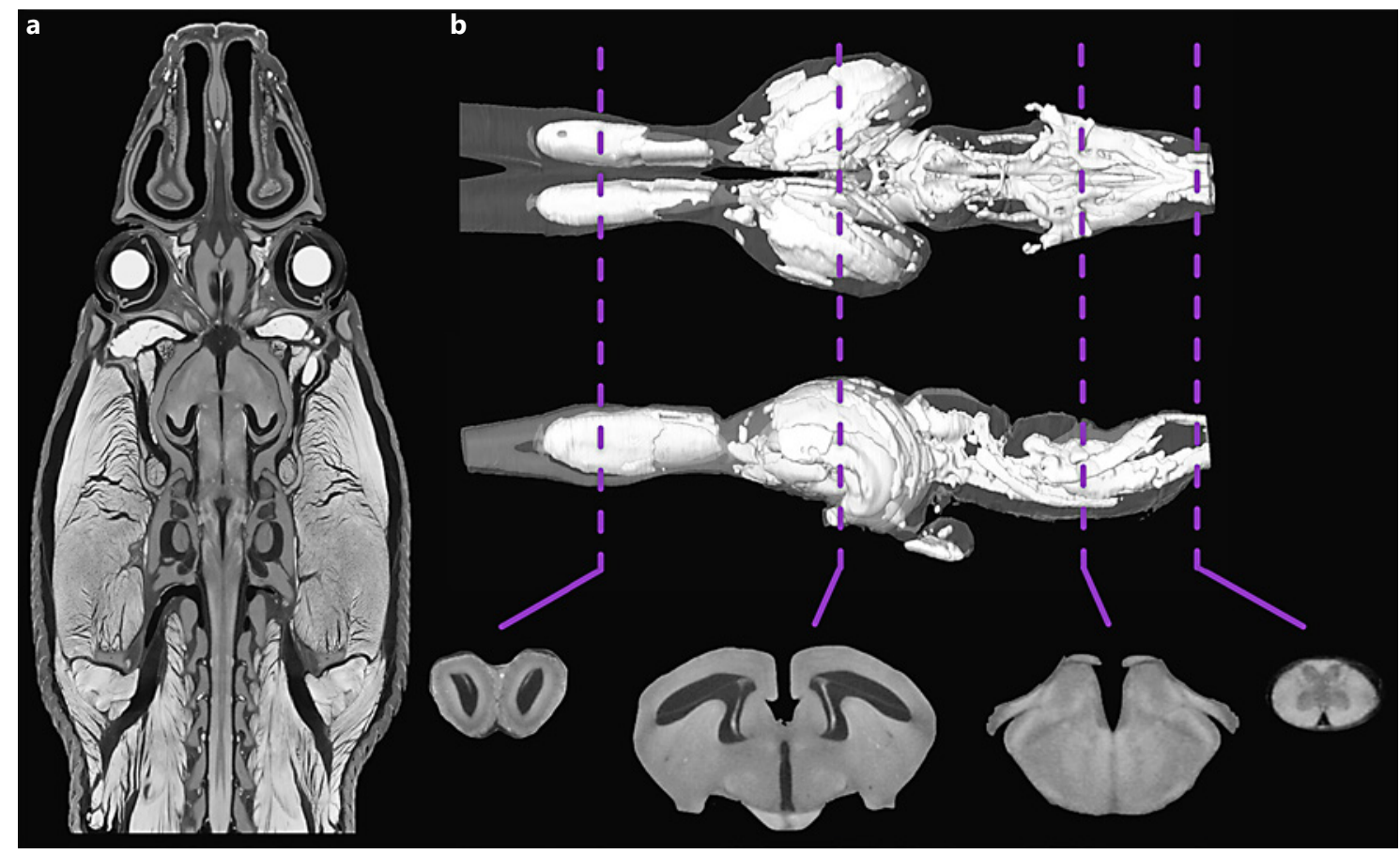

Fig. 4. DiceCT-based imaging and modeling of the brain and anterior spinal cord of an adult python (Liasis mackloti). a Frontal slice through the head and anterior-most portion of the trunk, showing the brain and spinal cord in situ within the skull and vertebral canal, respectively. (Note: The most radiodense structures appear as whiter in diceCT images, whereas relatively low-density structures appear as dark gray.) b 3D digital models of white-matter tracts (opaque white) and surrounding gray matter (semitrans- parent gray) in dorsal (above) and left lateral (below) projections. Bottom row of $2 \mathrm{D}$ transverse slices pass through (from left to right) the olfactory tract, forebrain, and midbrain at the optic chiasm (visible inferiorly), hind brain at the roots of the vestibulocochlear nerves (visible in the transverse section dorsolaterally, and in the frontal view in a), and the anterior-most portion of the spinal cord. (Preservation, staining, and $\mu \mathrm{CT}$-imaging parameters are available in online suppl. Table S4.) the scans of such specimens to exhibit a visual noise artifact as a result of X-ray attenuation. Complex neurological structures such as the brain house clusters of myelinated and unmyelinated neurons and nonneuronal cells that are packed closely together, which can cause them to take on a "noisy" appearance naturally because each stained cell type has a distinctly different X-ray attenuation coefficient. As a result, it can be particularly difficult to distinguish the edges of white-matter structures within the brain, for example, in short-duration diceCT scans. To overcome this issue, we recommend scanning specimens using protocols incorporating moderate to long Xray detector exposure timing (e.g., $\geq 333 \mathrm{~ms}$ ) and multiframe image averaging (e.g., 2-12×) [Degenhardt et al., 2010; Tahara and Larson, 2013; Cox and Faulkes, 2014; Gignac and Kley, 2014; Kleinteich et al., 2014]. Analogous to the functioning of an optical camera, higher exposure timing allows for the $\mathrm{X}$-ray detector to collect more $\mathrm{X}$ rays passing through the specimen. Multiframe averaging collects X-ray density data several times for the specimen in one rotation position or "step," and averages these independent samples. Together, this approach collects substantially more image data (X-rays), which produces specimens with sharper tissue boundaries and smoother gray-scale value $(\mathrm{GV})$ transitions within regions of the same tissue.

Figure 4 shows an example of this approach, using an $\mathrm{I}_{2}$ KI-stained Macklot's python (Liasis mackloti) head. The specimen was decapitated, fixed immediately in $10 \%$ neutral buffered formalin, and stained for 3 weeks in 5\% $\mathrm{I}_{2} \mathrm{KI}$. After staining, excess unbound iodine was washed out by immersion under running tap water for $\sim 24 \mathrm{~h}$. It was $\mu \mathrm{CT}$ scanned at an isometric voxel size of $20.5 \mu \mathrm{m}$, $140 \mathrm{kV}$, and $150 \mu \mathrm{A}$ (i.e., $21 \mathrm{~W}$ ), using a 0.1 -mm copper filter and a tungsten target in a GE phoenix v|tome|x s240 high-resolution microfocus CT system (General Electric, Fairfield, CT, USA). To achieve the fine level of detail within the brain and along cranial nerves, the X-ray de- 
tector was activated for 6 repeated 333-ms exposures (i.e., $1,998 \mathrm{~ms}$ of combined X-ray detection per rotation step), and all 6 exposures were averaged into a single density sample. This was repeated for each of 1,800 rotation steps across $360^{\circ}$ of X-ray sampling. This combination of high resolution and extended image timing required $>9 \mathrm{~h}$ of active beam time. (Note: The specimen was thoroughly wrapped in plastic to prevent desiccation.) Although this is a substantial period of scanning, the results illustrate how even subtle white-matter tracts within the brain can be readily visualized (Fig. 4; see online suppl. Videos S1-S3). Similar approaches were used for specimens in Figures 1-3 (see online suppl. Table S4).

\section{Data Processing}

DiceCT datasets are inherently complex. Detailed, 3D anatomy is represented initially by a continuous image stack (e.g., DICOM or TIFF files, most commonly). Depending on the size and resolution of the dataset, individual specimens may generate a few hundred megabytes to a few gigabytes of image data. However, individual datasets $>10 \mathrm{~GB}$, even for neurological regions of interest, are not uncommon. Determining what software resources have the greatest efficacy with diceCT data reflects a combination of image manipulation features, costs, and personal preferences. We use several software applications for data manipulation, 3D model building, and subsequent model processing. These are ImageJ (National Institutes of Health, Bethesda, MD), Avizo ${ }^{\odot}$ (Thermo Fisher Scientific, Waltham, MA, USA), and MeshLab (GNU General Public License), respectively. However, numerous alternative software packages are also available.

Data Manipulation. Image $\mathrm{J}$ is a free image and image stack processing tool. It is particularly adept at adjusting brightness and contrast to further enhance tissue differences in postprocessing, as well as rotating, cropping, translating, and reslicing entire image stacks. It provides for 3D manipulation of $\mu \mathrm{CT}$ data in a two-dimensional (2D) interface. We recommend ImageJ as a "go-to" tool for organizing diceCT data into standard orthogonal planes, which allows for easily evaluating the staining quality of the specimen, assessing right-left asymmetries, and exporting "fly-through" videos that are compressed and repackaged datasets, which are easier to share digitally (see online suppl. Videos S1-S3). (Documentation and tutorials are available at https://imagej.nih.gov/ij/ index.html.)

3D Model Building. There are several freely (e.g., Blender) and commercially (e.g., Avizo ${ }^{\odot}$ and VGStudio $\mathrm{Max}^{\odot}$ ) available digital reconstruction platforms that al- low image stacks to be represented and manipulated as $3 \mathrm{D}$ volumes. These platforms allow the user to select and add a portion of the total volume to user-defined digital "structures." This can be done manually (by selecting voxels with mouse clicks) or through automated means (by choosing a range of voxels with specific GVs, wherever they are located within the volume). In our experience with diceCT datasets, high-GV white matter is easily distinguished from lower-GV gray matter. In particular, white matter seems to present across the "whiter" range of GVs, which can be binned generally as low, medium, and high with respect to relative brightness. (Note: Most industrial-scale $\mu \mathrm{CT}$ scanners have cone-shaped beams, a geometry that precludes quantifying absolute GVs as is typical for medical CT scanners, which utilize parallel beams and output Hounsfield units [Gignac et al., 2016].) Within a given specimen, these differences are inferred to reflect variation in the extent of myelination, with exceptionally bright structures like thick optic nerves and chiasmata showing the highest GVs, and thin whitematter tracts appearing fainter (Fig. 4). 3D digital rendering programs allow the user to select narrow ranges of GVs for addition to digital structures, and an iterative approach that includes isolating low-, medium-, and then high-value GVs makes it fairly straightforward to select and render white-matter tracts within the brain (Fig. 4b; see online suppl. Surface S6) or extracranial peripheral nerve processes in isolated regions of interest (Fig. 3). Final, manual "cleanup" work is always recommended, including rendering remaining brain tissue as gray matter (online suppl. Surface S7) and having a specialist evaluate the neural reconstructions of greatest concern.

Model Processing. After producing 3D models and exporting them as surface files (e.g., stereolithography [*.STL], polygon [*.PLY], or object [*.OBJ] format), the resulting models will often be too large for easy sharing. However, the Quadric Edge Collapse Decimation tool in the freely available program MeshLab allows for broad surface smoothing that reduces surface file size without substantially altering the overall geometry or removing important neuroanatomical features (online suppl. Surfaces S6, S7). (Documentation and tutorials are available at http://www.meshlab.net.)

\section{Destaining and Reciprocal Illumination}

As discussed by Gignac et al. [2016], iodine staining can be reversed. Thus, one of the advantages of using iodine over other contrast agents is the potential for returning a vouchered specimen to a museum collection unstained. Alternatively, a destained diceCT specimen can 
be resampled for subsequent anatomical preparations (e.g., histological sectioning). Destaining can be achieved through progressive leaching of triiodide out of specimen tissues (i.e., placing the specimen in baths of water, formalin, ethanol, or other "clean" storage fluid, iteratively, until the staining agent ceases to diffuse out of the specimen and into solution). This process can take weeks to months, and it physically removes iodine from the specimen. Alternatively, one or more baths of a low concentration $(1-3 \% \mathrm{w} / \mathrm{v})$ of sodium thiosulfate $\left(\mathrm{Na}_{2} \mathrm{~S}_{2} \mathrm{O}_{3}\right)$ can be used to elicit an oxidation-reduction reaction with triiodide that transforms it into a colorless (and radiolucent) form of iodide $\left(\mathrm{I}^{-}\right)$[Trevorrow and Fashena, 1935; Shakhashiri, 1983; Kondo et al., 2001]. This process takes minutes to days, but it leaves the specimen forever chemically altered because iodide - although colorless - still remains within its tissues. Moreover, users should be aware that sodium thiosulfate is a chelating agent, and its use on vertebrate specimens may result in the decalcification of elements of the bony skeleton [A.C. Morhardt, pers. commun.; Pasch et al., 2008]. Although this side effect may be undesirable in certain circumstances, it does not interfere with subsequent histological protocols, which typically include thorough decalcification of specimens prior to sectioning [Humason, 1972].

Regardless of the destaining method, specimens can be reused subsequently in additional staining protocols. As demonstrated by Herdina et al. [2015b], using a bat phallus, destained specimens are amenable to subsequent staining under standard histological protocols (in this case, with Giemsa surface stain). Likewise, Hughes et al. [2016a, 2017] extended this approach for neural imaging with Nissl staining, using chameleon brains that had been previously stained for diceCT imaging but then subsequently destained. This work demonstrates the potential for pairing the gross morphology of the brain's white- and gray-matter regions (via diceCT) with cellular-level imaging in those regions for the same individual using standard histological approaches. There is immediate potential, therefore, for diceCT to be inserted into already established brain imaging workflows to reinforce or expand the value of data from each research specimen [Striedter et al., 2014].

\section{Community Development}

Over the last several years, a community of diceCT practitioners with various research foci has improved diceCT protocols and consolidated around recommend- ed best practices based on collective experience [Gignac et al., 2016]. To help ensure the rapid distribution of such advances, community members maintain diceCT.com as a centralized information hub for diceCT research and are active on social media (e.g., @diceCT on Twitter). Here, new users will find a regularly updated repository of diceCT publications, new research features, preprints of technique advances, and details of ongoing diceCT community events such as workshops, short courses, and conference symposia.

DiceCT is a widely applicable, rapid, and anatomically comprehensive new tool for generating high-resolution, 3D neuroanatomical datasets, which can be easily shared digitally with collaborators, publishers, and the general public. The removal/reversibility of the staining agent and potential to pair diceCT datasets with conventional histological methods will help bridge the wealth of published neural imaging data already available to approaches that consider the central and peripheral nervous systems as key aspects of highly integrated and complex phenotypes in 3D. We look forward to the continued advance of diceCT as updated approaches, drawn from experiences within the neuroanatomical sciences community, bring about high-resolution 3D brain imaging at increasingly finer scales.

\section{Acknowledgments}

We thank the J.B. Johnston Club for Evolutionary Neuroscience and Karger Workshop organizers for assembling this special issue, as well as A. Morhardt for inviting us to participate. We thank the Microscopy and Imaging Facility at the American Museum of Natural History (New York, NY, USA) and the Dentsply R\&D Office (Tulsa, OK, USA) for access to $\mu$ CT scanners. The Austin Working Group Meeting (University of Texas at Austin; April 2015) developed the best practices for diceCT. For conceptual and technical support, we thank M. Hill, K. Miller, H. O'Brien, A. Smith, K. Smith, H. Towbin, A. Ward, and the State Anatomical Board of Oklahoma. For providing access to facilities and/or specimens, we thank K. Ackerly, A. Balanoff, E. Ferrer, M. Gold, M. Norell, A. Turner, and A. Watanabe. We also thank the reviewers of an earlier version of this manuscript for the insights and constructive criticisms they provided. This work was supported financially by the National Science Foundation (NSF EAGER 1450842 and NSF IOS-0749750 awarded to N.J.K.; NSF EAGER 1450850 and NSF DEB 1457180 awarded to P.M.G.) as well as the Stony Brook University Department of Anatomical Sciences (N.J.K. and P.M.G.) and the Oklahoma State University Center for Health Sciences Department of Anatomy and Cell Biology (P.M.G.).
Gignac/Kley 


\section{Disclosure Statement}

The authors have no conflicts of interest to declare.

\section{Author Contributions}

Both authors conceived the project; analyzed and interpreted the data; wrote the paper; and created the figures.

\section{References}

Carlisle A, Selwood L, Hinds LA, Saunders N, Habgood M, Mardon K, Weisbecker V (2017): Testing hypotheses of developmental constraints on mammalian brain partition evolution, using marsupials. Sci Rep 7:4241.

Chung K, Wallace J, Kim S-Y, Kalyanasundaram S, Andalman AS, Davidson TJ, Mirzabekov JJ, Zalocusky KA, Mattis J, Denisin AK, Pak S, Bernstein H, Ramakrishnan C, Grosenick L, Gradinaru V, Deisseroth K (2013): Structural and molecular interrogation of intact biological systems. Nature 497:332-337.

Cox PG, Faulkes CG (2014): Digital dissection of the masticatory muscles of the naked molerat, Heterocephalus glaber (Mammalia, Rodentia). PeerJ 2:e448.

Cox PG, Jeffery N (2011): Reviewing the morphology of the jaw-closing musculature in squirrels, rats, and guinea pigs with contrastenhanced micro-CT. Anat Rec 294:915-928.

de Crespigny A, Bou-Reslan H, Nishimura MC, Phillips H, Carano RA, D’Arceuil HE (2008): $3 \mathrm{D}$ micro-CT imaging of the postmortem brain. J Neurosci Methods 171:207-213.

Degenhardt K, Wright AC, Horng D, Padmanabhan A, Epstein JA (2010): Rapid 3D phenotyping of cardiovascular development in mouse embryos by micro-CT with iodine staining. Circ Cardiovasc Imaging 3:314-322.

George ID, Holliday CM (2013): Trigeminal nerve morphology in Alligator mississippiensis and its significance for crocodyliform facial sensation and evolution. Anat Rec 296: 670-680.

Gignac PM, Kley NJ (2014): Iodine-enhanced micro-CT imaging: methodological refinements for the study of soft-tissue anatomy of postembryonic vertebrates. J Exp Zool B Mol Dev Evol 322:166-176.

Gignac PM, Kley NK, Clarke JA, Colbert MW, Morhardt AC, Cerio D, Cost IN, Cox PG, Daza JD, Early CM, Echols MS, Henkelman RM, Herdina AN, Holliday CM, Li Z, Mahlow K, Merchant S, Müller J, Orsbon CP, Paluh DJ, Thies ML, Tsai HP, Witmer LM (2016): Diffusible iodine-based contrast-enhanced computed tomography (diceCT): an emerging tool for rapid, high-resolution, 3-D imaging of metazoan soft tissues. J Anat 228:889909 .

Gold MEL, Shulz D, Budassi M, Vaska P, Gignac PM, Norell MA (2016): Flying starlings and PET lend insights on the evolution of volant dinosaurs. Curr Biol 26: R265-R267.
Herdina AN, Herzig-Straschil B, Hilgers $H$, Metscher BD, Plenk H Jr (2010): Histomorphology of the penis bone (baculum) in the gray long-eared bat Pleocotus austriacus (Chiroptera, Vespertilionidae). Anat Rec 293: 1248-1258.

Herdina AN, Kelly DA, Jahelkova H, Lina PHC, Horáček I, Metscher BD (2015a): Testing hypotheses of bat baculum function with $3 \mathrm{D}$ models derived from microCT. J Anat 226: 229-235.

Herdina AN, Plenk H Jr, Benda P, Lina PHC, Herzig-Straschil B, Hilgers $\mathrm{H}$, Metscher BD (2015b): Correlative 3D-imaging of Pipistrellus penis micromorphology: validating quantitative microCT images with undecalcified serial ground section histomorphology. J Morphol 276:695-706.

Holliday CM, Tsai HP, Skiljan RJ, George ID, Pathan S (2013): A 3D interactive model and atlas of the jaw musculature of Alligator mississippiensis. PLoS One 8:e62806.

Hughes DF, Gignac PM, Greenbaum E, Khan AM (2016a): Documenting brain diversity in fieldcaught lizards, from skull to cell: initial development of a processing pipeline for top down, multi-scale structural analyses of a single brain by integrating specialized microcomputed tomography (diceCT), Nissl-based cytoarchitectonics, and immunohistochemistry (abstract 778.02). Annu Meet Soc Neurosci.

Hughes DF, Gignac PM, Greenbaum E, Khan AM (2017): Don't forget the brain: elucidating the cranial diversity of Chamaeleonidae by incorporating validated field-based brain tissue preservation methods into multi-scale structural analyses (abstract). Joint Meet Ichthyologists Herpetologists.

Hughes DF, Gignac PM, Walker EM, Martinez A, Negishi K, Lieb CS, Greenbaum E, Khan AM (2016b): Rescuing perishable neuroanatomical information from a threatened biodiversity hotspot: remote field methods for brain tissue preservation validated by cytoarchitectonic analysis, immunohistochemistry, and iodine-enhanced X-ray microcomputed tomography. PLoS One 11:e0155824.

Humason GL (1972): Animal Tissue Techniques, ed 3. San Francisco, Freeman.

Jeffery GH, Bassett J, Mendham J, Denny RC (1989): Vogel's Textbook of Quantitative Chemical Analysis, ed 5. New York, Wiley.

Jeffery NS, Stephenson RS, Gallagher JA, Jarvis JC, Cox PG (2011): Micro-computed tomography with iodine staining resolves the arrangement of muscle fibres. J Biomech 44: 189-192.
Ketcham RA, Carlson WD (2001): Acquisition, optimization and interpretation of X-ray computed tomographic imagery: applications to the geosciences. Comput Geosci 27:381400 .

Kleinteich T, Conway KW, Gorb SN, Summers AP (2014): What's inside a fishy suction cup (abstract)? Bruker microCT User Meet.

Kondo H, Fukuda H, Ono H, Gotoda T, Saito D, Takahiro K, Shirao K, Yamaguchi H, Yoshida S (2001): Sodium thiosulfate solution spray for relief of irritation caused by Lugol's stain in chromoendoscopy. Gastrointest Endosc 53:199-202.

Lüsebrink F, Sciarra A, Mattern H, Yakupov R, Speck O (2017): T1-weighted in vivo human whole brain MRI dataset with an ultrahigh isotropic resolution of $250 \mu \mathrm{m}$. Sci Data 4: 170032.

Metscher BD (2009a): MicroCT for comparative morphology: simple staining methods allow high-contrast 3D imaging of diverse nonmineralized animal tissues. BMC Physiol 9: 11.

Metscher BD (2009b): MicroCT for developmental biology: a versatile tool for high-contrast 3D imaging at histological resolutions. Dev Dyn 238:632-640.

Metscher BD (2013): Biological applications of $\mathrm{X}$-ray microtomography: imaging microanatomy, molecular expression and organismal diversity. Microsc Anal 27:13-16.

Neu CP, Genin GM (2014): Handbook of Imaging in Biological Mechanics. Boca Raton, CRC Press.

Pasch A, Schaffner T, Huynh-Do U, Frey BM, Frey FJ, Farese S (2008): Sodium thiosulfate prevents vascular calcifications in uremic rats. Kidney Int 74:1444-1453.

Porro LB, Richards CT (2017): Digital dissection of the model organism Xenopus laevis using contrast-enhanced computed tomography. J Anat 231:169-191.

Schneider JE, Böse J, Bamforth SD, Gruber AD, Broadbent C, Clarke K, Neubauer S, Lengeling A, Bhattacharya S (2004): Identification of cardiac malformations in mice lacking Ptdsr using a novel high-throughput magnetic resonance imaging technique. BMC Dev Biol 4:16.

Shakhashiri B (1983): Chemical Demonstrations: A Handbook for Teachers of Chemistry. Madison, University of Wisconsin Press, vol 3 . 
Striedter GF, Belgard TG, Chen C-C, Davis FP, Finlay BL, Güntürkün $\mathrm{O}$, Hale $\mathrm{M}$, Harris JA, Hecht EE, Hof PR, Hofmann HA, Holland LZ, Iwaniuk AN, Jarvis ED, Karten HJ, Katz PS, Kristan WB, Macagno ER, Mitra PP, Moroz LL, Preuss TM, Ragsdale CW, Sherwood CC, Stevens CF, Stüttgen MK, Tsumoto T, Wilczynkski W (2014): NSF workshop report: discovering general principles of nervous system organization by comparing brain maps across species. Brain Behav Evol 83:1-8.

Tahara R, Larson HCE (2013): Quantitative analysis of microscopic X-ray computed tomography imaging: Japanese quail embryonic soft tissue with iodine staining. J Anat 223:297310.
Tobita K, Liu X, Lo CW (2010): Imaging modalities to assess structural birth defects in mutant mouse models. Birth Defects Res C Embryo Today 90:176-184.

Trevorrow V, Fashena G (1935): The determination of iodine in biological material. J Biol Chem 110:29-38.

Tsai HP, Holliday CM (2011): Ontogeny of the alligator cartilago transiliens and its significance for sauropsid jaw muscle evolution. PLoS One 6:e24935.

van de Moortele PF, Auerbach EJ, Olman C, Yacoub E, Uğurbil K, Moeller S (2009): T1 weighted brain images at 7 Tesla unbiased for Proton Density, T2* contrast and RF coil receive B1 sensitivity with simultaneous vessel visualization. NeuroImage 46:432-446.
Vickerton P, Jarvis J, Jeffery NS (2013): Concentration-dependent specimen shrinkage in iodine-enhanced microCT. J Anat 223:185193

Wallingford VH (1953): The development of organic iodine compounds as X-ray contrast media. J Am Pharm Assoc 42:721-728.

Webb WR, Brant W, Major N (2005): Fundamentals of Body CT, ed 3. Philadelphia, Elsevier.

Wong MD, Spring S, Henkelman RM (2013): Structural stabilization of tissue for embryo phenotyping using micro-CT with iodine staining. PLoS One 8:e84321. 